\title{
AVALIAÇÃO DE CUIDADOS NA TERAPIA INTRAVENOSA: DESAFIO PARA A QUALIDADE NA ENFERMAGEM ${ }^{\mathrm{a}}$
}

\author{
Evaluation of care in intravenous therapy: a challenge for quality in nursing \\ Evaluación de la atención en la terapia intravenosa: un desafío para la calidad en \\ enfermería
}

Ana Claudia Yassuko Murassaki ${ }^{1}$

Viviani Camboin Meireles ${ }^{4}$

\author{
Gelena Lucinéia Gomes da Silva Versa ${ }^{2}$ \\ Dagmar Willamowius Vituri ${ }^{5}$
}

José Aparecido Bellucci Júnior ${ }^{3}$

Laura Misue Matsuda ${ }^{6}$

\section{RESUMO}

Estudo multicêntrico, prospectivo, descritivo-exploratório, realizado em hospitais de ensino públicos (A e B), que objetivou avaliar cinco indicadores de qualidade de enfermagem relacionados à terapia intravenosa periférica. Utilizou-se o Instrumento de Registro de Busca Ativa em 1.307 observações dos seguintes indicadores: identificação e validade do acesso venoso periférico; de equipos; e dos frascos de soro. Determinou-se o Índice de Positividade (IP) para Qualidade da Assistência (QA): Desejável (100\% IP); Adequada (90-99\%); Segura (80-89\%); Limítrofe (71-79\%); Sofrível ( $\leq 70 \%)$. A QA apresentou-se Segura apenas para os indicadores identificação e validade de equipos e dos frascos de soro da instituição B. Os demais indicadores, em ambas as instituições, alcançaram QA Sofrível ou Limítrofe. Considerando que nenhum dos indicadores avaliados obteve QA Adequada nem Desejável, conclui-se que o processo de cuidado para com a terapia intravenosa, principalmente na instituição B, necessita de maior atenção por parte das lideranças e respectivas equipes.

Palavras-chave: Infusões intravenosas. Cuidados de enfermagem. Qualidade da Assistência à saúde. Enfermagem.

\begin{abstract}
Multicenter, prospective, descriptive and exploratory study, conducted in public teaching hospitals ( $\mathrm{A}$ and $\mathrm{B}$ ), which aimed to evaluate five nursing quality indicators related to peripheral intravenous therapy. It was used the Instrumento de Registro de Busca Ativa in 1307 observations of the following indicators: identification and validity of the peripheral venous access; from equipments and from serum bottles. It was determined the Positivity Index (PI) for Quality of Care (QC): Desirable (100\% PI) Adequate (90-99\%), Safe (80-89\%), Borderline (71-79\%), Low $(\leq 70 \%)$. The QC is presented Safe only for the indicators identification and validity of equipments and of serum bottles, from the institution $B$. The other indicators, at both institutions, reached Low or Borderline QC. Considering that none of the indicators evaluated reached Adequate QC, nor Desirable, it was concluded that the process of care for intravenous therapy, especially at institution $B$, requires more attention from leaders and their teams.
\end{abstract}

Keywords: Infusions Intravenous. Nursing Care. Quality of Health Care. Nursing.
Resumen

Estudio multicéntrico, prospectivo, descriptivo-exploratorio, realizado en hospitales de enseñanza públicos (A y B), cuyo objetivo fue evaluar cinco indicadores de calidad de enfermería relacionados con terapia intravenosa periférica. Se utilizó el Instrumento de Registro de Busca Ativa en 1307 observaciones de los siguientes indicadores: identificación y validez de la vía periférica venosa; equipos y frascos de suero. Se determinó el Índice de Positividad (IP) para Calidad de la Atención (CA): Deseable (100\% IP) Adecuada (90-99\%), Segura (80-89\%), Limítrofe (71-79\%), Baja ( $\leq 70 \%)$. La CA se presentó Segura apenas para los indicadores "identificación" y "validez" de equipos y de frascos de suero de la institución B. Los otros indicadores, en ambas instituciones, alcanzaron CA Baja o Limítrofe. Considerando que ningún de los indicadores evaluados logró CA Adecuada ni Deseable, se concluye que el proceso de atención a la terapia intravenosa, principalmente en la institución $B$, requiere más atención de los líderes y sus equipos.

Palabras clave: Infusiones Intravenosas. Atención de Enfermería. Calidad de la Atención de Salud. Enfermería.

\footnotetext{
${ }^{1}$ Enfermeira. Mestre em Enfermagem pela Universidade Estadual de Maringá. Bolsista da Coordenação de Aperfeiçoamento de Pessoal de Nível Superior. Maringá - PR. Brasil. E-mail: anamurassaki@yahoo.com.br; ²Enfermeira. Mestre em Enfermagem pela UEM. Maringá-PR. Enfermeira Intensivista da UTIadulto do Hospital Universitário do Oeste do Paraná - HUOP. Cascavel-PR. Brasil. E-mail: gelenaenfermagem@yahoo.com.br; ${ }^{3}$ Enfermeiro. Mestre em Enfermagem pela UEM. Maringá-PR. Docente do Setor de Enfermagem da Universidade Estadual do Norte do Paraná UENP. Bandeirantes-PR. E-mail: bellucci@uenp.edu.br; ㅌEnfermeira. Mestre em Enfermagem. Docente do Departamento de Enfermagem da Universidade Estadual de Maringá. Maringá

- PR. Brasil. E-mail: vcmeireles@ibest.com.br; ${ }^{5}$ Enfermeira Gerente de Risco do Hospital Universitário Regional do Norte, do Paraná, da Universidade Estadual de Londrina - HURNP - PR. Doutoranda em Ciências - Escola de Enfermagem de Ribeirão Preto - USP. Londrina - PR. Brasil. E-mail: dagmar@uel.br; ${ }^{6}$ Enfermeira. Doutora em Enfermagem. Docente do Departamento de Enfermagem da Universidade Estadual de Maringá - UEM. MaringáPR. Brasil. E-mail: Immatsuda@uem.br
} 


\section{INTRODUÇÃO}

$\mathrm{Na}$ atualidade, apesar de o desenvolvimento científico e tecnológico em todas as áreas do conhecimento, em especial na área da saúde, ter ocorrido em larga escala, os incidentes e eventos adversos se apresentam como grandes riscos à segurança do paciente e à qualidade do cuidado ${ }^{1}$. Como incidentes entendem-se as consequências do cuidado, que não causam lesões mensuráveis ou prolongamento do tempo de internação. Já os eventos adversos são ocorrências indesejáveis, porém evitáveis, de natureza iatrogênica, que causam danos mensuráveis e/ou prolongamento do tempo de internação e/ou óbito dos pacientes afetados ${ }^{1}$.

Nas instituições hospitalares, o envolvimento da equipe de enfermagem em eventos adversos relacionados à assistência ao paciente, em sua maioria, deve-se a erros de medicação; úlceras por pressão; quedas; flebites associadas a cateter venoso periférico; infecção no local da inserção do cateter venoso central; bacteremia relacionada à sonda vesical de demora, entre outros ${ }^{2}$. Pondera-se, ainda, que o erro pode estar relacionado a outros fatores, como a prática profissional, os produtos de saúde, os procedimentos e os sistemas ${ }^{3}$.

Sabe-se que, no sistema de medicação, todas as etapas e pessoas envolvidas são importantes. Apesar disso, este estudo enfocará unicamente atividades/indicadores pertinentes aos profissionais de enfermagem na terapia por infusão intravenosa, como a identificação e validade dos acessos venosos periféricos (AVP), dos equipos e dos frascos de soro.

Há de se destacar que complicações como flebite, infiltração/extravasamento, obstrução ou deslocamento acidental do dispositivo são descritas como eventos adversos relacionados ao AVP ${ }^{4}$, mas não serão enfocados neste estudo.

Considerando que a equipe de enfermagem é responsável pela inserção e manutenção do AVP, é premente que os cuidados técnicos recomendados sejam realizados a fim de se prevenir e/ou reduzir as iatrogenias relacionadas à instalação do dispositivo. Dentre os cuidados de enfermagem preconizados, destaca-se a troca do cateter em pacientes adultos no período de 72 a 96 horas $^{5}$, o que pressupõe a identificação correta e precisa do AVP.

Outro cuidado de enfermagem, não menos importante, que visa à prevenção de infecções refere-se à identificação e troca dos equipos de soro, os quais devem ser substituídos de acordo com o seu tipo e finalidade ${ }^{6}$, conforme prazos definidos pelo Center for Disease Control and Prevention.

No tocante à identificação dos rótulos de soro, fazse importante que esta seja realizada corretamente e de forma completa com a finalidade de se prevenir as iatrogenias mais comuns, quais sejam: omissão de doses; administração em concentração incorreta; aplicação em horários e vias impróprias; administração de medicamentos em pacientes trocados; ou ainda, administração errônea de fármacos ${ }^{2}$.

Os erros relacionados à medicação, além de comprometerem a qualidade do cuidado, impactam na hospitalização do paciente e geram ônus adicional à instituição ${ }^{7}$. Contudo, pode-se alcançar a segurança do paciente na terapia medicamentosa por intermédio de três ações interdependentes: evitar a ocorrência de eventos adversos, mas caso ocorram, torná-los visíveis e minimizar os seus efeitos ${ }^{8}$.

Com o intuito de obter informações que contribuam à segurança do paciente que faz uso de AVP, propõe-se a realização do presente estudo, pois a avaliação do sistema de medicação é uma ferramenta extremamente importante, não somente para o diagnóstico situacional do setor/serviço, mas como balizador de diretrizes de capacitação e gerenciamento da equipe de enfermagem, no concernente ao número e à qualificação profissional, voltado à garantia da segurança do paciente.

Mediante à problemática apresentada, estabeleceram-se os seguintes questionamentos: Os AVP, os frascos de soros e os equipos para infusão venosa são identificados e trocados conforme preconiza a literatura alusiva? A prática de enfermagem em terapia intravenosa periférica atende aos padrões de qualidade? Para responder a essas questões propõe-se a realização deste estudo, que tem como objetivo avaliar cinco indicadores de qualidade de cuidados de enfermagem relacionados à terapia intravenosa periférica.

\section{MÉTODOS}

Estudo multicêntrico, de caráter quantitativo, prospectivo, descritivo-exploratório, realizado em dois hospitais universitários públicos, aqui denominados de Hospital A e Hospital B, situados no interior paranaense. 0 primeiro hospital (A) dispõe de 30 leitos e o segundo (B) de 121 leitos, destinados à Clínica Médica e à Clínica Cirúrgica.

No Hospital A, participaram os pacientes/clientes de ambos os sexos, internados por, no mínimo, 24 horas, na Clínica Médica (CM) ou na Clínica Cirúrgica (CC). Para o Hospital B, adotaram-se os mesmos critérios, porém as Unidades são diferenciadas em Unidade Médica e Cirúrgica Feminina (UF) e Unidade Médica e Cirúrgica Masculina (UM).

A coleta de dados ocorreu no período de fevereiro de 2010 a abril de 2010, em seis dias aleatórios, durante três meses consecutivos, por meio de observação direta do paciente, utilizando-se de um formulário que avalia a qualidade dos cuidados de enfermagem, denominado 
"Instrumento de Registro de Busca Ativa", elaborado e validado por Vituri 9 .

0 instrumento antes referido é composto por 45 questões que avaliam 12 indicadores do cuidado de enfermagem: 1. Identificação do leito do paciente; 2. Identificação de risco para queda no leito; 3. Identificação de acessos venosos periféricos; 4. Verificação de lesões cutâneas pós-infiltrativas; 5. Identificação dos equipos para infusão venosa; 6 . Identificação dos frascos de soros e controle da velocidade de infusão; 7 . Identificação de sondas gástricas; 8. Fixação da sonda vesical de demora e posicionamento da bolsa coletora de diurese; 9. Checagem dos procedimentos da prescrição de enfermagem; 10. Controle de sinais vitais; 11. Checagem dos procedimentos de enfermagem na prescrição médica; 12. Elaboração da prescrição diária e completa pelo enfermeiro ${ }^{9}$.

0 foco do presente estudo circunscreve-se na avaliação das assertivas relacionadas à terapia intravenosa, quais sejam: Identificação do AVP; Validade do AVP; Identificação do equipo de infusão venosa; Validade do equipo para infusão venosa e; Identificação dos frascos de soro.

Concebeu-se como "Adequada" a identificação de AVP que, de acordo com a literatura ${ }^{6}$, contemplasse a data, a hora da inserção e a rubrica do funcionário responsável. Além disso, o prazo para a troca da punção obedeceu à recomendação do Centers for Disease Control and Prevention- $C D C^{5}$, que estabelece o período de $72 \mathrm{a}$ 96 horas.

Para a identificação do equipo de soro, considerou-se "Adequada" aquela que contivesse a data, a hora da troca e a rubrica do funcionário responsável pelo procedimento ${ }^{6}$. No que se refere ao prazo para troca, para a validade do equipo de soro considerou-se a determinação do $\mathrm{CDC}^{10}$, à época da coleta de dados, ou seja, 72 horas para equipos macrogotas e microgotas, com ou sem reservatório; 24 horas para equipos de pressão venosa central, nutrição parenteral e sangue / hemoderivados e 48 horas para equipo de bomba infusora. Ressalta-se, porém, que, na versão 2010 do CDC, o prazo para a troca dos equipos de macro e microgotas foi alterado para 96 horas a sete dias, desde que os dispositivos não tenham sido utilizados para sangue/derivados e soluções lipídicas ${ }^{11}$.

No que tange à identificação de rótulos de soro, utilizaram-se as recomendações estabelecidas pelo The National Coordinating Council for Medication Error Reporting and Prevention (NCC MERP) 3 e pela Resolução RDC 45/2003 - ANVISA, de 12 de março de 2003 que dispõe sobre o Regulamento Técnico de Boas Práticas de Utilização das Soluções Parenterais em Serviços de Saúde ${ }^{12}$. Assim, os rótulos deveriam conter os seguintes dados: nome do paciente; identificação do leito e da enfermaria; componentes da solução; volume, hora de início e de término previsto; tempo de infusão (número de gotas ou mililitros por hora); e assinatura do funcionário. A inexistência de qualquer um desses itens determinou a identificação como "Inadequada".

Os dados foram tabulados no Microsoft Office Excel $2007^{\circledR} \mathrm{e}$, para análise estatística descritiva, utilizou-se 0 software Statistic 8.0 $0^{\circledR}$.

Como padrão de qualidade, utilizaram-se os parâmetros adotados por Haddad ${ }^{13}$, cuja assistência de enfermagem é classificada a partir do Índice de Positividade (IP) de cada indicador avaliado.

A Qualidade da Assistência (QA) dos indicadores foi categorizada de acordo com o IP, conforme consta no Quadro 1.

Quadro 1. Critérios de classificação da qualidade da assistência de enfermagem.

\begin{tabular}{|c|c|}
\hline Qualidade da Assistência (QA) & Índice de Positividade (IP) \\
\hline Assistência desejável & $100 \%$ de positividade \\
\hline Assistência adequada & 90 a $99 \%$ de positividade \\
\hline Assistência segura & 80 a $89 \%$ de positividade \\
\hline Assistência limítrofe & 71 a $79 \%$ de positividade \\
\hline Assistência sofrível & $70 \%$ ou menos de positividade \\
\hline
\end{tabular}

Esta pesquisa obedeceu às exigências estabelecidas na Resolução 196/1996 ${ }^{14}$, com o parecer de aprovação n ${ }^{0}$. 482/2009 do Comitê Permanente de Ética em Pesquisas com Seres Humanos (COPEP), da Universidade Estadual de Maringá-PR.

\section{RESULTADOS}

Foram realizadas 374 observações no Hospital A e 933 no Hospital B, perfazendo um total de 1.307 coletas. No Quadro 2, constam os dados referentes aos cinco indicadores relacionados à terapia intravenosa periférica, assim como a classificação da QA obtida em cada unidade investigada.

Conforme se observa no Quadro 2, a QA foi classificada como Segura apenas na instituição B, para os indicadores Identificação de frasco de soro (UF e UM) e Identificação de equipo para infusão intravenosa (UM). Os demais indicadores obtiveram QA Sofríve/ nas unidades CM, CC e UF. 
Quadro 2. Qualidade daAssistência (QA) relacionada à administração de medicamentos em duas instituiçōes hospitalares de ensino do Paraná. Paraná, 2011.

\begin{tabular}{|c|c|c|c|c|c|c|}
\hline \multicolumn{2}{|l|}{ INDICADORES } & $\begin{array}{l}\text { Número de } \\
\text { observações }\end{array}$ & $\begin{array}{l}\text { Respostas } \\
\text { adequadas }\end{array}$ & $\begin{array}{c}\text { Respostas } \\
\text { inadequadas/ausentes }\end{array}$ & IP (\%) & $\overline{Q A}$ \\
\hline \multicolumn{7}{|c|}{ Identficaçāo de AVP } \\
\hline \multirow[t]{2}{*}{ Instituição A } & CM & 173 & 86 & 87 & 49,71 & Sofrivel \\
\hline & $\mathrm{CC}$ & 151 & 51 & 100 & 33,77 & Sofrivel \\
\hline \multirow[t]{2}{*}{ Instituição B } & UF & 328 & 170 & 498 & 51,83 & Sofrivel \\
\hline & UM & 265 & 207 & 58 & 78,11 & Limítrofe \\
\hline \multicolumn{7}{|c|}{ Validade do AVP } \\
\hline \multirow[t]{2}{*}{ Instituição A } & CM & 173 & 80 & 93 & 46,24 & Sofrivel \\
\hline & $\mathrm{CC}$ & 151 & 48 & 103 & 31,79 & Sofrivel \\
\hline \multirow[t]{2}{*}{ Instituição B } & UF & 328 & 164 & 164 & 50,00 & Sofrivel \\
\hline & UM & 265 & 205 & 60 & 77,36 & Limítrofe \\
\hline \multicolumn{7}{|c|}{ Identficaçāo de equipo para infusāo venosa } \\
\hline \multirow[t]{2}{*}{ Instituição A } & $C M$ & 202 & 77 & 125 & 38,12 & Sofrivel \\
\hline & $\mathrm{CC}$ & 196 & 65 & 131 & 33,16 & Sofrivel \\
\hline \multirow[t]{2}{*}{ Instituição B } & UF & 379 & 228 & 151 & 60,16 & Sofrivel \\
\hline & UM & 328 & 268 & 60 & 81,71 & Segura \\
\hline \multicolumn{7}{|c|}{ Validade de equipo para infusāo venosa } \\
\hline \multirow[t]{2}{*}{ Instituição A } & $C M$ & 323 & 121 & 202 & 37,46 & Sofrivel \\
\hline & $\mathrm{CC}$ & 338 & 110 & 228 & 32,54 & Sofrivel \\
\hline \multirow[t]{2}{*}{ Instituição B } & UF & 497 & 293 & 204 & 58,95 & Sofrivel \\
\hline & UM & 404 & 325 & 79 & 80,45 & Segura \\
\hline \multicolumn{7}{|c|}{ Identficaçāo de frasco de soro } \\
\hline \multirow[t]{2}{*}{ Instituição A } & $C M$ & 193 & 56 & 171 & 29,01 & Sofrivel \\
\hline & $\mathrm{CC}$ & 207 & 64 & 143 & 30,92 & Sofrivel \\
\hline \multirow[t]{2}{*}{ Instituição B } & UF & 254 & 216 & 38 & 85,04 & Segura \\
\hline & UM & 334 & 281 & 53 & 84,13 & se \\
\hline
\end{tabular}

\section{DISCUSSÃO}

Ao examinar os dados referentes aos indicadores Identificação do AVPe Validade do AVP (Quadro 2), observa-se que nas duas instituições pesquisadas, o IP obtido indica que a QA está aquém do nível seguro, uma vez que na UM foi considerada Limitrofe e nas demais unidades (CM, CC e UF), Sofrivel.

A falta ou a inadequação na identificação do AVP pode gerar consequências graves, pois dificulta a precisão do tempo de permanência do cateter ${ }^{15}$ o que está diretamente associado à ocorrência de flebite, infiltração, extravasamento, desconexão e deslocamento do dispositivo ${ }^{4}$, colocando em risco a segurança do paciente e a qualidade do cuidado prestado.

No concernente ao tempo de validade, nota-se que em $420(45,8 \%)$ avaliações, isto é, aproximadamente metade das observações de ambas as instituições, o paciente estava exposto ao risco de infecção relacionado à permanência do AVP além do tempo recomendado. A literatura ${ }^{10}$ aponta que a incidência de tromboflebite e colonização bacteriana aumenta em cateteres com tempo de permanência superior a 72 horas, sem diferença substancial se comparado a 96 horas.

No que tange aos indicadores /dentificação do equipo para infusão venosa e Validade de equipo para infusão venosa, todas as unidades (CM, CC e UF) alcançaram nível Sofrível de QA, exceto a UM, a qual obteve nível Seguro.

A ausência ou identificação inadequada do equipo, para infusão venosa, impossibilita o profissional de enfermagem determinar e respeitar os prazos de troca recomendados pelo
$C D C^{5}$. Condutas desse tipo expõem os clientes a riscos de adquirir infecção associada, o que pode resultar em danos ao cliente, à família e à instituição, além de um maior tempo de permanência e, consequente, aumento nos custos da internação?

Os achados relacionados à inadequação da identificação e validade de equipo de soro, bem como identificação e validade do AVP apontam para a necessidade urgente de estratégias de educação continuada e permanente em todas as unidades, a fim de eliminar fatores que aumentam o risco de erros. Além disso, faz-se mister o monitoramento sistemático da identificação e troca dos equipos de soro, bem como do sistema de medicação em geral, para que possíveis causas de erros sejam detectadas e corrigidas no intuito de garantir a qualidade do cuidado e, consequentemente, a segurança do paciente ${ }^{16}$.

Em relação ao indicador Identificação dos frascos de soro, os dados do Quadro 2 indicam que na Instituição B (UFe UM) a QA foi qualificada como Segura, enquanto na Instituição A (CM e CC) foi Sofrível.

0 resultado Sofrível da instituição merece atenção por parte das lideranças porque, à administração segura de infusões venosas contínuas, é imprescindível que o rótulo contenha todas as informações que possibilitem identificar o responsável pelo preparo, hora de início e término previsto, gotas ou mililitros por minuto e os componentes da solução com seus respectivos volumes ${ }^{3}$.

É importante ressaltar que, além de todas as informações referidas, é necessária a presença, no rótulo, da assinatura do profissional que realizou a medicação, para que seja possível apurar responsabilidades caso o paciente 
apresente alguma intercorrência relacionada à administração de medicamento ${ }^{9}$. Se o problema caracterizar ato de negligência ou imprudência por parte do profissional de enfermagem, este poderá ser identificado com segurança e responsabilizado ética e legalmente, conforme prevê a legislaçãa ${ }^{17}$.

Em que pese à necessidade de identificar a responsabilidade do profissional pelo erro de medicação, há que se considerar que o indivíduo raramente é a única causa. Para tanto, é preciso se atentar para as falhas do sistema de medicação, que é um processo complexo, multifatorial e multidisciplinar ${ }^{18}$, o qual deve ser estruturado com o escopo de propiciar condições que previnam ou minimizem os erros, por meio de estratégias como a notificação destes com vistas ao aprendizado e não à punição ${ }^{7}$.

Naturalmente que a conduta antes referida exige mudanças na forma de pensar e agir das lideranças, pois, de acordo com a abordagem da Qualidade, as não conformidades, na maioria das vezes, ocorrem devido a erro no processo e não às pessoas.

Ao ponderar sobre o IP obtido no Hospital A, em contraste com o Hospital $B$, referente à identificação do rótulo de soro, a divergência encontrada nos dois hospitais pode estar relacionada ao fato de que, no Hospital B, nos setores investigados, são realizadas avaliações periódicas pela Assessoria de Controle da Qualidade da Assistência de Enfermagem (ACQAE), com 0 mesmo instrumento utilizado neste estudo, o que possibilita intervenções imediatas junto à equipe. Assim, o Hospital B, possivelmente obteve IP mais elevado, porque o monitoramento de tais indicadores já faz parte da rotina do Serviço.

É importante destacar que, na maioria das vezes, os erros relacionados à medicação poderiam ser evitados se os enfermeiros estivessem menos ocupados com problemas burocráticos e administrativos e mais atuantes na supervisão direta do processo de medicação, que geralmente é realizado por profissionais de enfermagem de nível médio ${ }^{15}$.

A supervisão direta do enfermeiro corrobora para uma barreira defensiva no processo de medicação, reduzindo possíveis erros, uma vez que esse profissional direciona, orienta e avalia a equipe de enfermagem, ou seja, fornece condições favoráveis para o desenvolvimento de um cuidado de qualidade, livre de danos ou prejuízos ao paciente ${ }^{7}$.

No que se refere às estratégias para se evitar erros no processo de medicação, diversos métodos podem e devem ser adotados, como, por exemplo, a adesão dos profissionais às políticas e aos procedimentos que visem à segurança do cliente; acesso à informação; educação continuada e permanente; tecnologias e ambiente favorável para a minimização do erro e dimensionamento adequado de pessoal no trabalho ${ }^{16,18}$.

Outra estratégia, não menos importante, é aquela que contempla a participação do paciente em sua terapia medicamentosa e que deve ser incentivada por meio de políticas e diretrizes institucionais. Assim, é possível manter o paciente atualizado sobre os medicamentos que recebe e permitir que ele questione e alerte a equipe de saúde quando os medicamentos e/ou procedimentos não estiverem $\operatorname{corretos}^{18}$. A adoção da prática mencionada pela enfermagem é, sem dúvida, louvável porque permite a atuação pró-ativa por meio da identificação dos erros antes que estes ocorram.

No processo de atenção à saúde, o monitoramento e a avaliação das atividades concernentes à terapia intravenosa periférica são extremamente importantes, mas, conforme se observa neste estudo, é possível que essa prática não seja realizada sistematicamente no Hospital A e careça de investimentos no sentido de se tornar mais efetiva no Hospital B.

\section{CONCLUSÃO}

De acordo com os resultados, o cuidado de enfermagem relacionado à segurança do paciente no sistema de terapia intravenosa é ainda um grande desafio nas instituições estudadas, pois a maioria dos indicadores avaliados não atingiu o IP que caracteriza uma assistência de enfermagem Segura e de qualidade.

Perante a situação observada, fazem-se necessários investimentos em ações voltadas à educação continuada e permanente para a enfermagem das unidades estudadas, direcionadas à administração segura de medicamentos, em especial à terapia intravenosa. Além disso, outras ações devem ser tomadas no sentido de se estabelecer barreiras defensivas para se evitar os erros de medicação, tais como o incentivo à notificação dos erros; acesso fácil à informação; dimensionamento adequado da equipe; políticas e diretrizes institucionais que visem à segurança do paciente; participação do cliente no processo de terapia medicamentosa e supervisão direta e frequente da equipe pelo enfermeiro, nos processos de administração de medicamentos.

Reconhece-se que o presente estudo tem limitações por não ter abordado variáveis relacionadas ao pessoal de enfermagem e às taxas de ocupação dos hospitais investigados. Desse modo, sugerem-se estudos futuros que contemplem essas variáveis na avaliação da qualidade do cuidado de enfermagem, relacionado à terapia intravenosa.

Conclui-se que, apesar de os setores do Hospital B terem alcançado melhores resultados, a qualidade do cuidado de enfermagem em relação à terapia intravenosa necessita de maior atenção por parte das lideranças e de suas respectivas equipes, visto que nenhum dos indicadores avaliados neste estudo obteve QA Desejável (100\%) e nem Adequada (90-99\%). 


\section{REFERÊNCIAS}

1. Paiva MC, PaivaSA, Berti HW. Eventos adversos: análise de um instrumento de notificação utilizado no gerenciamento de enfermagem. Rev. Esc. Enferm. USP. 2010 jun; 44(2): 287-94.

2. Santos JC, Ceolim MF. latrogenias de enfermagem em pacientes idosos hospitalizados. Rev. Esc. Enferm. USP. 2009 dez; 43(4): 810-7.

3. National Coordinating Council for Medication Error Reporting and Prevention. NCC MERP Taxonomy of medication errors [citado em 2010 nov 03]. Disponível em: < http://www.nccmerp.org/pdf/taxo2001-0731.pdf>.

4. Batalha LMC, Costa LPS, Almeida DMG, Lourenço PAA, Gonçalves AMFM, Teixeira ACG. Fixação de cateteres venosos periféricos em crianças: estudo comparativo. Esc Anna Nery. 2010 jul/set; 14(3): 511-8.

5. Center for Disease Control and Prevention, Department of Health and Human Services. Guideline for the Prevention of Intravascular CatheterRelated Bloodstream Infections Final Issue Review. 2010 maio 17 [citado em 2011 fev 04]. Disponível em: <http://www.cdc.gov/hicpac/pdf/ BSI_guideline_IssuesMay17final.pdf $>$.

6. Banton J, Brady C, O’Kelley SD. Terapia intravenosa periférica. In: .Terapia intravenosa. Gomes IL, tradutor. Rio de Janeiro: Guanabara Koogan; 2005. p.17-49.

7. Teixeira TC, Cassiani SH. Análise de causa raiz: avaliação de erros de medicação em um hospital universitário. Rev. Esc. Enferm. USP. 2010 mar; 44(1): 139-46.

8. World Health Organization (WHO). Quality of care: patient safety. $55^{\circ}$ World Health Assembly. 2002 may [13-18]; Geneva(SUI): World Health Organization; 2002.

9. Vituri DW, Matsuda LM. Content validation of quality indicators for nursing care evaluation. Rev. Esc. Enferm. USP. 2009 jun; 43(2): 429-37.

10. O'Grady NP, et al. Guidelines for the Prevention of Intravascular Catheterrelated Infections. Clin. infect. dis. 2011; 52(9): 162-93.

11. O'Grady NP et al. Guidelines for the Prevention of Intravascular CatheterRelated Infections. MMWR: Morbidity And Mortality Weekly Report, Atlanta, v. 51, n. RR10, p. 1-26, 2002. Disponível em: < http://www.cdc.gov/ mmwr/preview/mmwrhtml/rr5110a1.htm\#top $>$.

12. Agência Nacional de Vigilância Sanitária (Brasil). Resolução RDC 45/ 2003 - ANVISA, de 12 de março de 2003 - Dispõe sobre o Regulamento Técnico de Boas Práticas de Utilização das Soluções Parenterais (SP) em Serviços de Saúde. Diário da República Federativa do Brasil, Brasilia(DF), 12 de março de 2003.

13. Haddad MCFL. Qualidade da assistência de enfermagem: o processo de avaliação em hospital público [tese de doutorado]. Ribeirão Preto (SP): Escola de Enfermagem de Ribeirão Preto/USP; 2004.

14. Conselho Nacional de Saúde (BR). Resolução n 196/96. Sobre Pesquisa Envolvendo Seres Humanos. Brasília(DF): Bioética; 1996.
15. Vituri DW, Cacciari P, Gvodz R, Kuwabara CCT, Cardoso MGP. Indicadores de qualidade como estratégia para melhoria da qualidade do cuidado em um hospital universitário. Ciênc. cuid. saúde. 2010 out/dez; 9(4): 78290.

16. Wannmacher L. Erros: evitar o evitável. [internet]. 2005 jun; 2(7): 16. (Uso racional de medicamentos: temas selecionados, 7)

17. Conselho Federal de Enfermagem. Resolução COFEN n. 311/2007 Código de Ética dos Profissionais de Enfermagem [citado em 2010 out 28]. Disponível em: <http://site.portalcofen.gov.br/node/4394>.

18. Cassiani SHB, Miasso Al, Silva AEBC, Fakin FT, Oliveira RC. Aspectos gerais e número de etapas do sistema de medicação de quatro hospitais brasileiros. Rev. Latino-Am. Enfermagem. 2004 set/out; 12(5): 781-9.

\section{NOTA}

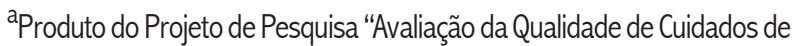
Enfermagem em Instituições de Ensino Público" subsidiado pelo Conselho Nacional de Desenvolvimento Tecnológico e Científico - CNPq. 